Original Article Journal of Epilepsy Research pISSN 2233-6249 / eISSN 2233-6257

Received November 30, 2016 Accepted March 3, 2017

Corresponding author: Asie Shojaii Research Institute for Islamic and Complementary Medicine and School of Traditional Medicine, Iran University of Medical Sciences, Tehran, Iran

Tel. +98-21-55152191

Fax. +98-21-55613191

E-mail; Shojaii.a@iums.ac.ir

\section{Anticonvulsant Effects of the Hydroalcoholic Extract of Alpinia officinarum Rhizomesin Mice: Involvement of Benzodiazepine and Opioid Receptors}

\author{
Shaghayegh Rezvani Nejad ${ }^{1}$, Manijeh Motevalian ${ }^{1}$, Iman Fatemi ${ }^{2}$, Asie Shojaii ${ }^{3}$ \\ ${ }^{1}$ Department of Pharmacology, School of Medicine, Iran University of Medical Sciences, Tehran; ${ }^{2}$ Department of \\ Physiology and Pharmacology, Physiology-Pharmacology Research Center, Rafsanjan University of Medical \\ Sciences, Rafsanjan; ${ }^{3}$ Research Institute for Islamic and Complementary Medicine and School of Traditional Medicine, \\ Iran University of Medical Sciences, Tehran, Iran
}

\begin{abstract}
Background and Purpose: Epilepsy is one of the most common serious neurological conditions. The current therapeutic treatment of epilepsy with modern antiepileptic drugs is associated with side effects, dose-related and chronic toxicity, and teratogenic effects and in approximately $30 \%$ of the patients is ineffective. Alpinia officinarum is used in Iranian traditional medicine for treatment of different diseases like back pain and seizure.

Methods: In this study, anticonvulsant effects of hydroalcoholic extract of Alpinia officinarum rhizomes were examined by using pentylentetrazole (PTZ) model in mice. Alpinia officinarum rhizomes extract (200, 400 and $600 \mathrm{mg} / \mathrm{kg}$ ), diazepam (1 mg/kg) and normal saline $(10 \mathrm{~mL} / \mathrm{kg}$ ) were injected (ip) 30 minutes before PTZ (90 mg/kg, ip). The time taken before the onset of clonic convulsions, the duration of colonic convulsions, and the percentage of seizure and mortality protection were recorded. For further clarification of the mechanism of action for Alpinia officinarum, flumazenil ( $2 \mathrm{mg} / \mathrm{kg}$, ip) and naloxone ( $5 \mathrm{mg} / \mathrm{kg}$, ip) were also injected 5 minutes before Alpinia officinarum extract.
\end{abstract}

Results: Alpinia officinarum extract at the doses of 200 and $400 \mathrm{mg} / \mathrm{kg}$ prolonged the time of onset of seizure and decreased the duration of seizures compared to control (saline) group $(p<0.05)$. At the dose of $600 \mathrm{mg} / \mathrm{kg}$, percentage of seizure protection was $16.66 \%$. Naloxone and flumazenil could suppress anticonvulsant effects of Alpinia officinarum.

Conclusions: It seems that Alpinia officinarum could be a good candidate and be useful for seizure control and treatment, and in these effects, opioid and benzodiazepine receptors might probably be involved.

(2017;7:33-38)

Key words: Alpinia officinarum, Seizure, Hydroalcoholic extract, Naloxone, Flumazenil

\section{Introduction}

Epilepsy is one of the most common neurological disorders characterized by spontaneous and recurrent seizures. Seizures are caused by abnormal and excessive discharges produced by a group of cerebral neurons. ${ }^{1}$ It is demonstrated that repeated and prolonged seizures produce mental (cognitive and behavioral) impairments via induction of neuronal death. ${ }^{2}$ Epilepsy decreases the quality of life and increases the risk of injury and even mortality. ${ }^{3}$ Despite new developments in antiepileptic drugs, there are difficulties in cure of the different types of epilepsy. ${ }^{4}$ In addition, about one-third of patients are considered to be pharmaco-resistant and do not respond to these medications. ${ }^{5}$ Therefore, it is necessary to explore new pharmacological treatments; especially those originated from plants and other natural sources. ${ }^{6-8}$

Alpinia officinarum (Zingiberaceae), commonly known as galangal, is one of the medicinal plants recommended for treatment of seizure in Iranian traditional medicine. ${ }^{9}$ Some other pharmacological activities also have been mentioned in traditional medicine for various conditions such as abdominal pain, emesis, diarrhea, impotence, renal diseases, and dysentery. ${ }^{10}$ Recent studies have demonstrated that A/pinia officinarum extracts possess a wide variety of biological activ- 
ities including antioxidant, ${ }^{11}$ anti-inflammatory, ${ }^{12}$ immunostimulatory, ${ }^{13}$ and anticancer ${ }^{14}$ activities. Several bioactive components of A/pinia officinarum have been reported in the literature such as diaryl heptanoids, flavonoids and essential oils. ${ }^{15}$

According to our knowledge, some traditional uses of Alpinia officinarum have been confirmed in modern scientific studies. But the antiepileptic effect of $A /$ pinia officinarum has not been studied so far. Following our previous studies on antiepileptic effect of Iranian medicinal plant, ${ }^{7}$ in the present study, anticonvulsant activity and possible mechanisms of action of the rhizomes of A/pinia officinarum hydroalcoholic extract (AOE) were studied in pentylenetetrazole induced seizures in mice for the first time.

\section{Methods}

\section{Preparation of the hydroalcoholic extract of $A /$ pinia officinarum rhizome}

Alpinia officinarum rhizomes were purchased from a local herbal market in Tehran, Iran and its authenticity was approved by Dr. Golamreza Amin (School of Pharmacy, Tehran University of Medical Sciences, Tehran, Iran). The voucher specimen number PMP-234 was deposited in the school of pharmacy. Hydroalcoholic extract (70:30 alcohol:water) of A/pinia officinarum rhizome was prepared by maceration method (3 times) and the extract was dried by rotary evaporator and stored at $-20^{\circ} \mathrm{C}$ until tested. The extract was dissolved in normal saline when used in the animal study.

\section{Animals}

In this study, male albino mice weighing 25-35 g (animal house of Iran University of Medical Sciences, Tehran, Iran) were used. The animals were housed in polycarbonate cages under $24 \pm 2^{\circ} \mathrm{C}$ with a 12-hour light/dark cycles. All animals were similarly handled in the animal house and had free access to food and tap water. All possible steps were taken to avoid animal suffering at each stage of the experiment. The ethical guidelines for using experimental animals were followed in all tests in accordance with ethical committee acts of Iran University of Medical Sciences and the European Communities Council Directive 24 November 1986 (86/609/EEC).

\section{Chemicals}

Drugs used in this study are as follows: pentylenetetrazole (PTZ) (Sigma, USA), flumazenil (Sigma, USA), diazepam (Sigma, USA), naloxone (Sigma, USA). All drugs were dissolved in sterile physiological saline.

\section{Experimental studies}

\section{Anticonvulsant activity}

PTZ (60 mg/kg, i.p.) was used to induce convulsions in the mice. ${ }^{16}$ The animals were divided randomly into 5 groups $(n=6)$ as follows: 1) control group, received normal saline (10 mL/kg, i.p.) $30 \mathrm{~min}$ before administrating PTZ; 2) diazepam group, received diazepam (1 mg/kg, i.p.) 30 min before administrating PTZ; 3-5) AOE groups, received different doses of AOE dissolved in water $(200,400$ and $600 \mathrm{mg} / \mathrm{kg}$, i.p.) $30 \mathrm{~min}$ before administrating PTZ. Following induction of convulsions in mice, the seizure parameters were immediately documented. Each animal was placed into an individual plastic cage and monitored for $30 \mathrm{~min}$ for signs of neurological deficits, particularly hindlimb tonic extensions. The seizure latency, seizure duration and percentage of seizure protection were recorded and the animals were observed for mortality for $24 \mathrm{~h}$ after PTZ injection.

\section{Evaluation of the effect of flumazenil on the anticonvu-}

\section{Isant activity of $\mathrm{AOE}$}

To investigate the possible involvement of benzodiazepine receptors, we examined the effects of flumazenil (benzodiazepine receptor antagonist) on the anticonvulsant activity of $\mathrm{AOE} .{ }^{17}$ The animals were divided randomly into 6 groups $(n=6)$ as follows: 1$)$ control group, received normal saline (10 mL/kg, i.p.) 30 min before administrating PTZ; 2) flumazenil group, received flumazenil (2 mg/kg, i.p.) 20 min before administrating PTZ; 3) diazepam group, received diazepam (1 mg/kg, i.p.) 30 min before administrating PTZ; 4) Diazepam + Flumazenil group, received flumazenil ( $2 \mathrm{mg} / \mathrm{kg}$, i.p.) $5 \mathrm{~min}$ before the injection of diazepam ( $1 \mathrm{mg} / \mathrm{kg}$, i.p.) and $20 \mathrm{~min}$ before the injection of PTZ; 5) AOE group, received AOE $(600 \mathrm{mg} / \mathrm{kg}$, i.p.) 30 min before administrating PTZ; 6) AOE 600 + flumazenil group, received flumazenil ( $2 \mathrm{mg} / \mathrm{kg}$, i.p.) $5 \mathrm{~min}$ before the injection of AOE (600 mg/kg, i.p.) and 20 min before the injection of PTZ. ${ }^{17}$ Then seizure latency, seizure duration and percentage of seizure protection in 30 min after injection of PTZ, and also mortality rate during the first $24 \mathrm{~h}$ were recorded. The anticonvulsant activity of AOE and diazepam in mice pretreated with flumazenil was assessed and compared with mice in control, flumazenil, diazepam and AOE (600 $\mathrm{mg} / \mathrm{kg}$, i.p.) groups.

Evaluation of the effect of naloxone on the anticonvulsant activity of $\mathrm{AOE}$

To investigate the probable modulatory effect of opioid receptors, we examine the effects of naloxone (opioid receptor antagonist) on 
the anticonvulsant activity of AOE. ${ }^{18}$ The animals were divided randomly into 4 groups $(n=6)$ as follows: 1$)$ control group, received normal saline (10 mL/kg, i.p.) 30 min before administrating PTZ; 2) naloxone group, received naloxone ( $5 \mathrm{mg} / \mathrm{kg}$, i.p.) $20 \mathrm{~min}$ before administrating PTZ; 3) AOE group, received AOE (600 mg/kg, i.p.) 30 min before administrating PTZ; 4) AOE 600 + naloxone group, received naloxone $(5 \mathrm{mg} / \mathrm{kg}$, i.p.) $5 \mathrm{~min}$ before the injection of $\mathrm{AOE}$ ( $600 \mathrm{mg} / \mathrm{kg}$, i.p.) and $20 \mathrm{~min}$ before the injection of PTZ. ${ }^{18}$ Then seizure latency, seizure duration and percentage of seizure protection in 30 min after injection of PTZ, and also mortality rate during the first $24 \mathrm{~h}$ were recorded. The anticonvulsant activity of AOE in mice pretreated with naloxone was assessed and compared with mice in control, naloxone and AOE (600 mg/kg, i.p.) groups.

\section{Statistical analysis}

The results of the study are reported as mean \pm S.E.M. The differences among the groups were evaluated by one-way analysis of variance followed by the Tukey test by using GraphPad Prism 5.0 (GraphPad Software Inc., La Jolla, CA, USA) software. For all tests, results with $p<0.05$ were considered as statistically significant.

\section{Results}

\section{Anticonvulsant activity of AOE on PTZ-induced seizure}

Injection of the AOE (400 and $600 \mathrm{mg} / \mathrm{kg}$ ) prolonged significantly the onset of seizure ( $p<0.05$ and $p<0.001$, respectively) and decreased the duration of seizures ( $p<0.05$ and $p<0.01$, respectively) compared with the control group. In addition, AOE (600 mg/kg) exhibited protection against seizure in 100\% of animals and reduced mortality compared with the control group. Diazepam protected $100 \%$ of mice from PTZ-induced seizure and mortality (Table 1).

\section{The effect of flumazenil on the anticonvulsant activity of $A O E$}

Administration of flumazenil 5 min before AOE $(600 \mathrm{mg} / \mathrm{kg})$ and 20 min before the injection of PTZ, resulted in inhibition of prolonged latency produced by AOE $(p<0.001)$; but had no significant effect on duration of seizure. However, the difference between the latency and the duration of seizure in mice that had received AOE $(600 \mathrm{mg} / \mathrm{kg}$ ) pretreated with flumazenil and the control group (all $p<0.01)$ was statistically significant. Also, Flumazenil reduced seizure protection (\%) in AOE (600 mg/kg) from $100 \%$ to $16.66 \%$. So, flumazenil reversed the anticonvulsant action of AOE to some extent. In addition, flumazenil inhibited the anticonvulsant activity of diazepam (Table 2).

Table 1. Effect of $A O E$ in PTZ-induced seizures in mice

\begin{tabular}{lcccc}
\hline Treatment group & \% Protection $(30 \mathrm{~min})$ & Seizure latency $(\mathrm{s})$ & Seizure duration $(\mathrm{s})$ & Mortality $(24 \mathrm{~h})$ \\
\hline Control & 0 & $87.80 \pm 8.434$ & $58.00 \pm 15.14$ & $4 / 6$ \\
Diazepam & 100 & $\infty^{\star * *}$ & $0.00 \pm 0.00^{* *}$ & $0 / 6$ \\
AOE 200 & 0 & $147.3 \pm 62.34$ & $23.00 \pm 5.508$ & $2 / 6$ \\
AOE 400 & 0 & $751.3 \pm 319.7^{*}$ & $18.67 \pm 8.353^{*}$ & $0 / 6$ \\
AOE 600 & 100 & $\infty^{\star * *}$ & $0.00 \pm 0.00^{* *}$ & $0 / 6$ \\
\hline
\end{tabular}

The data are expressed as mean \pm S.E.M. $(n=6)$.

$\mathrm{AOE}$, Alpinia officinarum extrac; PTZ, pentylenetetrazole.

${ }^{*} p<0.05 ;{ }^{* *} p<0.01 ;{ }^{* *} p<0.001$ compared to the control group.

Table 2. Effect of flumazenil on the anticonvulsant activity of AOE and diazepam in PTZ-induced convulsion in mice

\begin{tabular}{lcccc}
\hline Treatment group & \% Protection $(30 \mathrm{~min})$ & Seizure latency $(\mathrm{s})$ & Seizure duration $(\mathrm{s})$ & Mortality $(24 \mathrm{~h})$ \\
\hline Control & 0 & $87.80 \pm 8.434$ & $58.00 \pm 15.14$ & $4 / 6$ \\
Flumazenil & 0 & $77.33 \pm 3.844$ & $52.00 \pm 6.083$ & $3 / 6$ \\
Diazepam & 100 & $\infty^{\star * *}$ & $0.00 \pm 0.00^{* *}$ & $0 / 6$ \\
Diazepam + Flumazenil & 0 & $113.7 \pm 10.34$ & $54.33 \pm 7.535$ & $2 / 6$ \\
AOE 600 & 100 & $\infty^{\star * *}$ & $0.00 \pm 0.00^{* *}$ & $0 / 6$ \\
AOE 600 + Flumazenil & 16.66 & $739.5 \pm 58.7 * * \# \#$ & $11.75 \pm 4.973^{* *}$ & $1 / 6$ \\
\hline
\end{tabular}

The data are expressed as mean \pm S.E.M. $(n=6)$. Abbreviations: PTZ, pentylenetetrazole; AOE, Alpinia officinarum extract. ${ }^{*} p<0.05 ;{ }^{* *} p<0.01 ;{ }^{* *} p<0.001$ compared to the control group; ${ }^{\# \#} p<0.001$ compared to the AOE 600 group. 
Table 3. Effect of naloxone on the anticonvulsant activity of AOE in in PTZ-induced convulsion in mice

\begin{tabular}{lcccc}
\hline Treatment group & \% Protection $(30 \mathrm{~min})$ & Seizure latency $(\mathrm{s})$ & Seizure duration $(\mathrm{s})$ & Mortality $(24 \mathrm{~h})$ \\
\hline Control & 0 & $87.80 \pm 8.434$ & $58.00 \pm 15.14$ & $4 / 6$ \\
Naloxone & 0 & $53.40 \pm 3.763$ & $50.20 \pm 4.259$ & $4 / 6$ \\
AOE 600 & 100 & $\infty^{* * *}$ & $0.00 \pm 0.00^{* *}$ & $0 / 6$ \\
AOE $600+$ naloxone & 16.66 & $630.8 \pm 394.8^{\# \#}$ & $20.50 \pm 9.251^{*}$ & $1 / 6$ \\
\hline
\end{tabular}

The data are expressed as mean \pm S.E.M. $(n=6)$.

PTZ, pentylenetetrazole; AOE, Alpinia officinarum extract.

${ }^{\star} p<0.05 ;{ }^{* *} p<0.01 ;{ }^{* * *} p<0.001$ compared to the control group; ${ }^{\# \#} p<0.01$ compared to the AOE 600 group.

\section{The effect of naloxone on the anticonvulsant activity of $\mathrm{AOE}$}

Administration of naloxone $5 \mathrm{~min}$ before $\mathrm{AOE}(600 \mathrm{mg} / \mathrm{kg})$ and 20 min before the injection of PTZ, resulted in inhibition of prolonged latency produced by AOE $(p<0.01)$; but had no significant effect on duration of seizure. However, the difference between the duration of seizure in mice that had received AOE $(600 \mathrm{mg} / \mathrm{kg})$ pretreated with naloxone and the control group $(p<0.05)$ was statistically significant. Also, Naloxone reduced seizure protection (\%) in AOE $(600 \mathrm{mg} / \mathrm{kg}$ ) from $100 \%$ to $16.66 \%$. So, naloxone reversed the anticonvulsant action of AOE to some extent (Table 3).

\section{Discussion}

The results of our study indicated that AOE significantly reduces the seizures duration and increases latency period of seizures induced by PTZ in mice in a dose-dependent manner. We also found that these effects may be related to its effect on opioid and benzodiazepines receptors since the effect was highly diminished by opioid) and benzodiazepine receptor antagonists.

A/pinia officinarum is originally from China and it has been reported to have an array of biological activities including antioxidant, anti-inflammatory, immunostimulatory, and anticancer clinical applications. ${ }^{19}$ Although A/pinia officinarum has been used traditionally for many centuries, a few studies were undertaken for evaluation of its safety and the systematic toxicity. In a recent study, Honmore et al., ${ }^{20}$ reported that oral administration of methanolic extract of A/pinia officinarum with $2000 \mathrm{mg} / \mathrm{kg}$ did not produce any behavioral abnormalities and mortality.

PTZ is a known chemoconvulsant model for studying epilepsy which causes the seizure similar to petit mal epilepsy. It is well-demonstrated that PTZ induces convulsions by inhibiting and/or attenuation of GABAergic neurotransmission. ${ }^{21}$ Anticonvulsant drugs like diazepam and phenobarbital treat seizure by inhibiting $G_{A B A_{A}}$ receptor. ${ }^{22}$ Several studies in human and animal have demonstrated the important role of oxidative stress in the pathophysiology of epilepsy. ${ }^{23}$ These studies showed that during seizure, lipid peroxidation and nitric oxide (NO) formation do increase, while antioxidant enzyme activities decrease. ${ }^{24}$ Moreover, it has been demonstrated that free radicals reduce GABAergic neurotransmission and $G A B A_{A}$ receptor is highly sensitive to oxidative stress. ${ }^{25}$ The anticonvulsant effects of antioxidant agents suggesting that oxidative stress may be implicated in epilepsy. ${ }^{26}$ Furthermore, it has been demonstrated that diazepam (as a prototype for anticonvulsant drugs) exert its anticonvulsant effects by direct enhancement of $G A B A_{A}$ receptor and decreasing NO formation. ${ }^{27}$ In the present study, we found that AOE (400 and $600 \mathrm{mg} / \mathrm{kg}$ ) injection prolonged the onset of seizure and decreased the duration of seizures compared with the control group. Our results also indicated that administration of flumazenil before AOE $(600 \mathrm{mg} / \mathrm{kg})$ antagonizes the effect of AOE on latency time seizures in the PTZ model. The results of the present study suggested that anticonvulsant effects of AOE might have been mediated directly and/or indirectly by acting on GABA complex, since benzodiazepines have receptor on GABA complex and we have shown that pretreatment with benzodiazepine antagonist, flumazenil, could prevent the effect of AOE on seizure. In addition, antioxidant effects of Alpinia officinarum are reported in previous studies. ${ }^{20,28}$ So, there is also a possibility that AOE exert its antiseizure effect via antioxidant activity. This needs further investigation.

It is well established that central opioidergic system possesses seizure-modulating properties. ${ }^{29}$ Several investigations suggest that low doses of morphine ( $\mu$-opioid receptor agonist) have an anticonvulsive effect, while in high doses, it increase the seizure susceptibility induced by chemoconvulsant drugs such as picrotoxin and PTZ. ${ }^{30}$ The relation between GABAergic system and central opioidergic system in seizure modulation has been shown previously. ${ }^{31}$ Accordingly, naloxone (a nonselective opioid receptor antagonist) inhibits these effects of morphine. ${ }^{32}$ We found that administration of naloxone before AOE $(600 \mathrm{mg} / \mathrm{kg})$ decreased the prolongation of seizure latency induced by AOE in the PTZ model. Hence, it seems 
that the anticonvulsive effects of $\mathrm{AOE}$ could have been mediated by the opioid system.

It is well established that the brain or peripheral tissue inflammation reduces the threshold for seizures. ${ }^{33}$ Various studies consistently demonstrated the important involvement of inflammatory mediators in pathogenesis of epilepsy. ${ }^{34}$ They showed that epilepsy increases the level of proinflammatory cytokines like NO, TNF- $\alpha$, IL- 6 and IL-8. ${ }^{35}$ In addition, some studies showed the important role of cyclooxygenase isoenzymes in the epilepsy. They have tested COX-inhibitors with promising results in the treatment of epilepsy. ${ }^{36}$ Several lines of evidence support the contention that AOE has profound anti-inflammatory effects. Mirzaei et al., ${ }^{37}$ showed that hydroalcoholic extract of A/pinia officinarum have anti-inflammatory effect via inhibition of NF- $\mathrm{\kappa B}$ signaling. NF- $\mathrm{\kappa B}$ regulates the transcription of a large number of genes, particularly those involved in the inflammatory responses such as cyclooxygenase-2 (COX-2), inducible nitric-oxide synthase (iNOS), TNF- $\alpha, \mathrm{IL}-6$ and IL-8. ${ }^{38}$ Moreover, Honmore et al., demonstrated that A/pinia officinarum had anti-inflammatory effects through the COX-inhibitory effects and was useful in treatment of inflammatory disorders. ${ }^{20}$ Accordingly, the anti-inflammatory effects of AOE may possibly inhibit the seizures induced by PTZ in mice.

Several constituents of Alpinia officinarum are known namely: methylcinnamate, $\alpha$-cadinene, apigenin, kaempferide, alpinin, galangol, and some diarylheptanoids like curcumin. Some of these active components have significant protective effect in different animal models of seizure. ${ }^{19}$ For example, in a recent study, Han et al. ${ }^{39}$ demonstrated that apigenin have anticonvulsant activity in kainate-induced seizure via antioxidative properties. The anti-epileptic action of curcumin was demonstrated in various studies. ${ }^{40,41}$ Accordingly, it might suggest that apigenin and curcumin present in in AOE could have been responsible for the anticonvulsant properties exhibited by hydroalcoholic extract of this plant.

In conclusion, results of our study showed the anticonvulsant activity of hydroalcoholic extract of A/pinia officinarum rhizomes. Also, our results suggest that benzodiazepines or/and opioidergic system could have been partially involved in the anticonvulsant activity of Alpinia officinarum. These results support the traditional use of the plant in the treatment of convulsion. However, further research is required to find out the underlying mechanism(s) and the active component(s) responsible for the observed activity.

\section{References}

1. Thurman DJ, Beghi E, Begley $C E$, et al. Standards for epidemiologic studies and surveillance of epilepsy. Epilepsia 2011;52 Suppl 7:2-26.

2. Kiasalari Z, Khalili M, Roghani M, Heidari H, Azizi Y. Antiepileptic and antioxidant effect of hydroalcoholic extract of ferula assa foetida gum on pentylentetrazole-induced kindling in male mice. Basic Clin Neurosci 2013:4:299-306.

3. Jalili C, Salahshoor M, Pourmotabbed A, et al. The effects of aqueous extract of Boswellia Serrata on hippocampal region CA1 and learning deficit in kindled rats. Res Pharm Sci 2014;9:351-8.

4. Sendrowski K, Sobaniec W. Hippocampus, hippocampal sclerosis and epilepsy. Pharmacol Rep 2013;65:555-65.

5. Bhutada $P$, Mundhada $Y$, Bansod K, Dixit P, Umathe S, Mundhada D. Anticonvulsant activity of berberine, an isoquinoline alkaloid in mice. Epilepsy Behav 2010;18:207-10.

6. Mehrzadi S, Shojaii A, Pur SA, Motevalian M. Anticonvulsant activity of hydroalcoholic extract of citrullus colocynthis fruit involvement of benzodiazepine and opioid receptors. J Evid Based Complementary Altern Med 2015:21(4):NP31-5.

7. Mehrzadi S, Sadr S, Hosseinzadeh A, et al. Anticonvulsant activity of the ethanolic extract of Punica granatum L. seed. Neurol Res 2015;37:470-5.

8. Rashidian A, Farhang $F$, Vahedi $H$, et al. Anticonvulsant effects of Lippia citriodora (Verbenaceae) leaves ethanolic extract in mice: role of gabaergic system. Int J Prev Med 2016;7:97.

9. Abdollahi Fard M, Shojaii A. Efficacy of Iranian traditional medicine in the treatment of epilepsy. Biomed Res Int 2013;2013:692751

10. Jung B, Shin M. Illustrated encyclopedia of Korean medicinal plants. Seoul: Younglim Press; 1990. p. 250-1.

11. Shi GF, An $\sqcup$, Jiang B, Guan S, Bao YM. Alpinia protocatechuic acid protects against oxidative damage in vitro and reduces oxidative stress in vivo. Neurosci Lett 2006;403:206-10.

12. Israf $D$, Khaizurin $T$, Syahida $A$, Lajis $N$, Khozirah $S$. Cardamonin inhibits COX and iNOS expression via inhibition of p65NF- $\kappa$ B nuclear translocation and $I_{\kappa}-B$ phosphorylation in RAW 264.7 macrophage cells. Mol Immunol 2007;44:673-9.

13. Bendjeddou D, Lalaoui K, Satta D. Immunostimulating activity of the hot water-soluble polysaccharide extracts of Anacyclus pyrethrum, Alpinia galanga and Citrullus colocynthis. I Ethnopharmacol 2003;88:155-60.

14. Lee C, Houghton P. Cytotoxicity of plants from Malaysia and Thailand used traditionally to treat cancer. J Ethnopharmaco/ 2005;100:237-43.

15. Shin D, Kinoshita K, Koyama K, Takahashi K. Antiemetic principles of Alpinia officinarum. J Nat Prod 2002;69:1315-8.1

16. Fatehi F, Fatemi I, Shamsizadeh A, et al. The effect of hydroalcoholic extract of Pistacia vera on pentylenetetrazole-induced kindling in rat. Res J Pharmacogn 2017:4:45-51.

17. Hosseinzadeh $\mathrm{H}$, Parvardeh $\mathrm{S}$. Anticonvulsant effects of thymoquinone, 
the major constituent of Nigella sativa seeds, in mice. Phytomedicine 2004; 11:56-64.

18. Kaminski RM, Witkin JM, Shippenberg TS. Pharmacological and genetic manipulation of kappa opioid receptors: Effects on cocaine-and pentylenetetrazol-induced convulsions and seizure kindling. Neuropharmacology 2007;52:895-903.

19. Jung $\mathrm{CH}$, Jang SJ, Ahn J, et al. Alpinia officinarum inhibits adipocyte differentiation and high-fat diet-induced obesity in mice through regulation of adipogenesis and lipogenesis. J Med Food 2012;15:959-67.

20. Honmore VS, Kandhare $A D$, Kadam PP, et al. Isolates of Alpinia officinarum Hance as COX-2 inhibitors: Evidence from anti-inflammatory, antioxidant and molecular docking studies. Int Immunopharmacol 2016:33:8-17.

21. Corda $\mathrm{M}$, Giorgi $\mathrm{O}$, Longoni $\mathrm{B}$, Orlandi M, Biggio $\mathrm{G}$. Decrease in the Function of the $\gamma$-Aminobutyric Acid-Coupled Chloride Channel Produced by the Repeated Administration of Pentylenetetrazol to Rats. J Neurochem 1990;55:1216-21.

22. Meldrum B. GABA-agonists as anti-epileptic agents. Adv Biochem Psychopharmacol 1981;26:207-17.

23. Aguiar $C C$, Almeida $A B$, Araújo PV, et al. Oxidative stress and epilepsy: literature review. Oxid Med Cell Longev 2012;2012:795259.

24. Bashkatova V, Narkevich V, Vitskova G, Vanin A. The influence of anticonvulsant and antioxidant drugs on nitric oxide level and lipid peroxidation in the rat brain during penthylenetetrazole-induced epileptiform model seizures. Prog Neuropsychopharmacol Biol Psychiatry . 2003;27:487-92.

25. Sah R, Galeffi F, Ahrens R, Jordan G, Schwartz-Bloom RD. Modulation of the $\mathrm{GABA}(\mathrm{A})$-gated chloride channel by reactive oxygen species. J neurochem 2002;80:383-91.

26. Tomé Ada R, Ferreira PM, Freitas RM. Inhibitory action of antioxidants (ascorbic acid or $\alpha$-tocopherol) on seizures and brain damage induced by pilocarpine in rats. Arqu Neuro-psiquiatr 2010;68: 355-61.

27. Fernández-Cancio $M$, Fernández-Vitos EM, Imperial $S$, Centelles JJ. Structural requirements of benzodiazepines for the inhibition of pig brain nitric oxide synthase. Brain Res Mol Brain Res 2001;96:87-93.

28. Heo MY, Sohn SJ, Au WW. Anti-genotoxicity of galangin as a cancer chemopreventive agent candidate. Mutat Res 2001;488:135-50.

29. Hong J. Hippocampal opioid peptides and seizures. Epilepsy Res Supp/
1991;7:187-95.

30. Foote F, Gale K. Proconvulsant effect of morphine on seizures induced by pentylenetetrazol in the rat. Eur J Pharmacol 1984;105:179-84.

31. Simmons ML, Chavkin C. Endogenous opioid regulation of hippocampal function. Int Rev Neurobiol 1996:39:145-96.

32. Lauretti GR, Ahmad I, Pleuvry B. The activity of opioid analgesics in seizure models utilizing N-methyl-DL-aspartic acid, kainic acid, bicuculline and pentylenetetrazole. Neuropharmacology 1994;33:155-60.

33. Walker L, Sills GJ. Inflammation and epilepsy: the foundations for a new therapeutic approach in epilepsy? Epilepsy Curr 2012;12:8-12.

34. Rao R, Medhi B, Saikia U, et al. Experimentally induced various inflammatory models and seizure: understanding the role of cytokine in rat. Eur Neuropsychopharmacol 2008;18:760-7.

35. Vezzani A, Balosso S, Ravizza T. The role of cytokines in the pathophysiology of epilepsy. Brain Behav Immun 2008;22:797-803.

36. Vieira V, Glassmann D, Marafon P, Pereira P, Gomez R, Coitinho AS. Effect of diclofenac sodium on seizures and inflammatory profile induced by kindling seizure model. Epilepsy Res 2016;127:107-13.

37. Mirzaei K, Hossein-nezhad A, Aslani S, Emamgholipour S, Karimi M, Keshavarz S. Alpinia Energy expenditure regulation via macrophage migration inhibitory factor in obesity and in vitro anti-macrophage migration inhibitory factor effect of Alpinia officinarum hance extraction. Endocr Pract 2012;18:39-48.

38. Lee J-H, Jung HS, Giang PM, et al. Blockade of nuclear factor- $\kappa$ B signaling pathway and anti-inflammatory activity of cardamomin, a chalcone analog from Alpinia conchiger. I Pharmacol Exp Ther 2006;316:271-8.

39. Han JY, Ahn SY, Kim CS, et al. Protection of apigenin against kainate-induced excitotoxicity by anti-oxidative effects. Bio PharmBull 2012;35:1440-6.

40. Zhu W, Su J, Liu J, Jiang C. The involvement of neuronal nitric oxide synthase in the anti-epileptic action of curcumin on pentylenetetrazol-kindled rats. Biomed Mater Eng 2015;26 Suppl 1:S841-50.

41. Sumanont $Y$, Murakami $Y$, Tohda $M$, Vajragupta $O$, Watanabe $H$, Matsumoto K. Prevention of kainic acid-induced changes in nitric oxide level and neuronal cell damage in the rat hippocampus by manganese complexes of curcumin and diacetylcurcumin. Life Sci 2006;78:1884-91. 